George A. Anastassiou (Memphis, TN)

IOANNIS K. Argyros (Lawton, OK)

\title{
A CONVERGENCE ANALYSIS FOR EXTENDED ITERATIVE ALGORITHMS WITH APPLICATIONS TO FRACTIONAL AND VECTOR CALCULUS
}

Abstract. We give local and semilocal convergence results for some iterative algorithms in order to approximate a locally unique solution of a nonlinear equation in a Banach space setting. In earlier studies the operator involved is assumed to be at least once Fréchet-differentiable. In the present study, we assume that the operator is only continuous. This way we extend the applicability of iterative algorithms. We also present some choices of the operators involved in fractional calculus and vector calculus where the operators satisfy the convergence conditions.

1. Introduction. In this study we are concerned with the problem of approximating a locally unique solution $x^{*}$ of the nonlinear equation

$$
F(x)=0,
$$

where $F$ is a continuous operator defined on a subset $D$ of a Banach space $X$ with values in a Banach space $Y$.

A lot of problems in computational sciences and other disciplines can be brought into the form (1.1) using mathematical modelling [7, 12, 16, 18, 19]. The solutions of such equations can be found in closed form only in special cases. That is why most solution methods for these equations are iterative. Iterative algorithms are usually studied based on semilocal and local convergence. The semilocal convergence analysis is, based on the information around the initial point, to give hypotheses ensuring the convergence of the

2010 Mathematics Subject Classification: 65G99, 65H10, 26A33, 47J25, 47J05.

Key words and phrases: iterative algorithm, Banach space, semilocal-local convergence, fractional calculus, generalized fractional derivatives, vector calculus.

Received 8 July 2015; revised 2 April 2017.

Published online 8 August 2017. 
iterative algorithm; while the local convergence analysis is, based on the information around a solution, to find estimates of the radii of convergence balls as well as error bounds on the distances involved.

We introduce the iterative algorithm defined for each $n=0,1,2, \ldots$ by

$$
x_{n+1}=x_{n}-\left(A(F)\left(x_{n}\right)\right)^{-1} F\left(x_{n}\right),
$$

where $x_{0} \in D$ is an initial point and $A(F)(x) \in L(X, Y)$ the space of bounded linear operators from $X$ into $Y$. There is a plethora of studies on local as well as semilocal convergence theorems for the iterative algorithm (1.2) provided that the operator $A$ is an approximation to the Fréchet derivative $F^{\prime}[1,2,5-17]$. In the present study we do not assume that $A$ is related to $F^{\prime}$. This way we extend the applicability of $(1.2)$. Notice that many well known methods are special cases of 1.2 :

Newton's method: Choose $A(F)(x)=F^{\prime}(x)$ for each $x \in D$.

Steffensen's method: Choose $A(F)(x)=[x, G(x) ; F]$, where $G: X \rightarrow X$ is a known operator and $[x, y ; F]$ denotes a divided difference of order one [7, 12, 15.

The so called Newton-like methods and many other methods are special cases of (1.2).

The rest of the paper is organized as follows. The semilocal and local convergence analysis of the iterative algorithm $(1.2)$ is given in Section 2 . Some applications from fractional calculus are given in Section 3.

2. Convergence analysis. We present the main semilocal convergence result for the iterative algorithm 1.2.

TheOrem 2.1. Let $F: D \subset X \rightarrow Y$ be a continuous operator and let $A(F)(x) \in L(X, Y)$. Suppose that there exist $x_{0} \in D, \eta \geq 0, p \geq 1$, and a function $h:[0, \eta] \rightarrow[0, \infty)$ continuous and nondecreasing such that for each $x, y \in D$,

$$
\begin{aligned}
& (A(F)(x))^{-1} \in L(Y, X), \\
& \left\|\left(A(F)\left(x_{0}\right)\right)^{-1} F\left(x_{0}\right)\right\| \leq \eta, \\
& \left\|(A(F)(y))^{-1}(F(y)-F(x)-A(F)(x)(y-x))\right\| \\
& \leq h(\|x-y\|)\|x-y\|^{p+1}, \\
& q:=h(\eta) \eta^{p}<1, \\
& \bar{U}\left(x_{0}, r\right) \subseteq D \text {, }
\end{aligned}
$$

where

$$
r=\frac{\eta}{1-q} .
$$


Then the sequence $\left\{x_{n}\right\}$ generated by 1.2 is well defined, remains in $\bar{U}\left(x_{0}, r\right)$ for each $n=0,1,2, \ldots$ and converges to some $x^{*} \in \bar{U}\left(x_{0}, r\right)$ such that

$$
\begin{aligned}
\left\|x_{n+1}-x_{n}\right\| & \leq h\left(\left\|x_{n}-x_{n-1}\right\|\right)\left\|x_{n}-x_{n-1}\right\|^{p+1} \leq q\left\|x_{n}-x_{n-1}\right\|, \\
\left\|x_{n}-x^{*}\right\| & \leq \frac{q^{n} \eta}{1-q} .
\end{aligned}
$$

Proof. The iterate $x_{1}$ is well defined by $(1.2)$ for $n=0$ and (2.1) for $x=x_{0}$. By (2.2) and (2.6) we also have $\left\|x_{1}-x_{0}\right\|=\left\|\left(A(F)\left(x_{0}\right)\right)^{-1} F\left(x_{0}\right)\right\| \leq$ $\eta<r$, so $x_{1} \in \bar{U}\left(x_{0}, r\right)$ and $x_{2}$ is well defined (by 2.5). Using (2.3) for $y=x_{1}, x=x_{0}$ and (2.4) we get

$$
\begin{aligned}
\left\|x_{2}-x_{1}\right\| & =\left\|\left(A(F)\left(x_{1}\right)\right)^{-1}\left[F\left(x_{1}\right)-F\left(x_{0}\right)-A(F)\left(x_{0}\right)\left(x_{1}-x_{0}\right)\right]\right\| \\
& \leq h\left(\left\|x_{1}-x_{0}\right\|\right)\left\|x_{1}-x_{0}\right\|^{p+1} \leq q\left\|x_{1}-x_{0}\right\|,
\end{aligned}
$$

which shows (2.7) for $n=1$. Then

$$
\begin{aligned}
\left\|x_{2}-x_{0}\right\| & \leq\left\|x_{2}-x_{1}\right\|+\left\|x_{1}-x_{0}\right\| \leq q\left\|x_{1}-x_{0}\right\|+\left\|x_{1}-x_{0}\right\| \\
& =(1+q)\left\|x_{1}-x_{0}\right\| \leq \frac{1-q^{2}}{1-q} \eta<r,
\end{aligned}
$$

so $x_{2} \in \bar{U}\left(x_{0}, r\right)$ and $x_{3}$ is well defined.

Assuming $\left\|x_{k+1}-x_{k}\right\| \leq q\left\|x_{k}-x_{k-1}\right\|$ and $x_{k+1} \in \bar{U}\left(x_{0}, r\right)$ for each $k=1, \ldots, n$ we get

$$
\begin{aligned}
\left\|x_{k+2}-x_{k+1}\right\| & =\left\|\left(A(F)\left(x_{k+1}\right)\right)^{-1}\left[F\left(x_{k+1}\right)-F\left(x_{k}\right)-A(F)\left(x_{k}\right)\left(x_{k+1}-x_{k}\right)\right]\right\| \\
& \leq h\left(\left\|x_{k+1}-x_{k}\right\|\right)\left\|x_{k+1}-x_{k}\right\|^{p+1} \\
& \leq h\left(\left\|x_{1}-x_{0}\right\|\right)\left\|x_{1}-x_{0}\right\|^{p}\left\|x_{k+1}-x_{k}\right\| \leq q\left\|x_{k+1}-x_{k}\right\|
\end{aligned}
$$

and

$$
\begin{aligned}
\left\|x_{k+2}-x_{0}\right\| & \leq\left\|x_{k+2}-x_{k+1}\right\|+\left\|x_{k+1}-x_{k}\right\|+\cdots+\left\|x_{1}-x_{0}\right\| \\
& \leq\left(q^{k+1}+q^{k}+\cdots+1\right)\left\|x_{1}-x_{0}\right\| \leq \frac{1-q^{k+2}}{1-q}\left\|x_{1}-x_{0}\right\| \\
& <\frac{\eta}{1-q}=r
\end{aligned}
$$

which completes the induction for 2.7$)$ and $x_{k+2} \in \bar{U}\left(x_{0}, r\right)$. We also have, for $m \geq 0$,

$$
\begin{aligned}
\left\|x_{n+m}-x_{n}\right\| & \leq\left\|x_{n+m}-x_{n+m-1}\right\|+\cdots+\left\|x_{n+1}-x_{n}\right\| \\
& \leq\left(q^{m-1}+q^{m-2}+\cdots+1\right)\left\|x_{n+1}-x_{n}\right\| \\
& \leq \frac{1-q^{m}}{1-q} q^{n}\left\|x_{1}-x_{0}\right\| .
\end{aligned}
$$

It follows that $\left\{x_{n}\right\}$ is a Cauchy sequence in the Banach space $X$ and as such it converges to some $x^{*} \in \bar{U}\left(x_{0}, r\right)$. By letting $m \rightarrow \infty$, we obtain 2.8.

Stronger hypotheses are needed to show that $x^{*}$ is a solution of $F(x)=0$. 
Proposition 2.2. Let $F: D \subset X \rightarrow Y$ be a continuous operator and let $A(F)(x) \in L(X, Y)$. Suppose that there exist $x_{0} \in D, \eta \geq 0, p \geq 1, \psi>0$, and a function $h_{1}:[0, \eta] \rightarrow[0, \infty)$ continuous and nondecreasing such that for each $x, y \in D$,

$$
\begin{aligned}
& A(F)(x)^{-1} \in L(Y, X), \\
& \left\|(A(F)(x))^{-1}\right\| \leq \psi, \quad\left\|\left(A(F)\left(x_{0}\right)\right)^{-1} F\left(x_{0}\right)\right\| \leq \eta, \\
& \|F(y)-F(x)-A(F)(x)(y-x)\| \leq \frac{h_{1}(\|x-y\|)}{\psi}\|x-y\|^{p+1}, \\
& q_{1}:=h_{1}(\eta) \eta^{p}<1, \\
& \bar{U}\left(x_{0}, r_{1}\right) \subseteq D,
\end{aligned}
$$

where

$$
r_{1}=\frac{\eta}{1-q_{1}} .
$$

Then the conclusions of Theorem 2.1 for $\left\{x_{n}\right\}$ hold with $h_{1}, q_{1}, r_{1}$ replacing $h, q$ and $r$, respectively. Moreover, $x^{*}$ is a solution of $F(x)=0$.

Proof. Notice that

$$
\begin{aligned}
&\left\|\left(A(F)\left(x_{n}\right)\right)^{-1}\left[F\left(x_{n}\right)-F\left(x_{n-1}\right)-A(F)\left(x_{n-1}\right)\left(x_{n}-x_{n-1}\right)\right]\right\| \\
& \leq\left\|\left(A(F)\left(x_{n}\right)\right)^{-1}\right\| F\left(x_{n}\right)-F\left(x_{n-1}\right)-A(F)\left(x_{n-1}\right)\left(x_{n}-x_{n-1}\right) \| \\
& \leq h_{1}\left(\left\|x_{n}-x_{n-1}\right\|\right)\left\|x_{n}-x_{n-1}\right\|^{p+1} \leq q_{1}\left\|x_{n}-x_{n-1}\right\| .
\end{aligned}
$$

Therefore, the proof of Theorem 2.1 applies. Then, in view of the estimate

$$
\begin{aligned}
\left\|F\left(x_{n}\right)\right\| & =\left\|F\left(x_{n}\right)-F\left(x_{n-1}\right)-A(F)\left(x_{n-1}\right)\left(x_{n}-x_{n-1}\right)\right\| \\
& \leq \frac{h_{1}\left(\left\|x_{n}-x_{n-1}\right\|\right)}{\psi}\left\|x_{n}-x_{n-1}\right\|^{p+1} \leq q_{1}\left\|x_{n}-x_{n-1}\right\|,
\end{aligned}
$$

we deduce by letting $n \rightarrow \infty$ that $F\left(x^{*}\right)=0$.

Concerning the uniqueness of the solution $x^{*}$ we have the following result:

Proposition 2.3. Under the hypotheses of Proposition 2.2, suppose further that

$$
q_{1} r_{1}^{p}<1
$$

Then $x^{*}$ is the only solution of $F(x)=0$ in $\bar{U}\left(x_{0}, r_{1}\right)$.

Proof. The existence of the solution $x^{*} \in \bar{U}\left(x_{0}, r_{1}\right)$ has been established in Proposition 2.2. Let $y^{*} \in \bar{U}\left(x_{0}, r_{1}\right)$ with $F\left(y^{*}\right)=0$. Then

$$
\begin{aligned}
\left\|x_{n+1}-y^{*}\right\| & =\left\|x_{n}-y^{*}-\left(A(F)\left(x_{n}\right)\right)^{-1} F\left(x_{n}\right)\right\| \\
& =\left\|\left(A(F)\left(x_{n}\right)\right)^{-1}\left[A(F)\left(x_{n}\right)\left(x_{n}-y^{*}\right)-F\left(x_{n}\right)+F\left(y^{*}\right)\right]\right\|
\end{aligned}
$$




$$
\begin{aligned}
& \leq\left\|\left(A(F)\left(x_{n}\right)\right)^{-1}\right\|\left\|F\left(y^{*}\right)-F\left(x_{n}\right)-A(F)\left(x_{n}\right)\left(y^{*}-x_{n}\right)\right\| \\
& \leq \psi \frac{h_{1}\left(\left\|x_{n}-y^{*}\right\|\right)}{\psi}\left\|x_{n}-y^{*}\right\|^{p+1} \leq q_{1} r_{1}^{p}\left\|x_{n}-x^{*}\right\|<\left\|x_{n}-y^{*}\right\|,
\end{aligned}
$$

so $\lim _{n \rightarrow \infty} x_{n}=y^{*}$. But we know that $\lim _{n \rightarrow \infty} x_{n}=x^{*}$. Hence, $x^{*}=y^{*}$.

Next, we present a local convergence analysis for 1.2 .

Proposition 2.4. Let $F: D \subset X \rightarrow Y$ be a continuous operator and let $A(F)(x) \in L(X, Y)$. Suppose that there exist $x^{*} \in D, p \geq 1$, and a function $h_{2}:[0, \infty) \rightarrow[0, \infty)$ continuous and nondecreasing such that for each $x \in D$,

$$
\begin{gathered}
F\left(x^{*}\right)=0, \quad(A(F)(x))^{-1} \in L(Y, X) \\
\left\|(A(F)(x))^{-1}\left[F(x)-F\left(x^{*}\right)-A(F)(x)\left(x-x^{*}\right)\right]\right\| \\
\leq h_{2}\left(\left\|x-x^{*}\right\|\right)\left\|x-x^{*}\right\|^{p+1}
\end{gathered}
$$

and $\bar{U}\left(x^{*}, r_{2}\right) \subseteq D$, where $r_{2}$ is the smallest positive solution of the equation

$$
h^{*}(t):=h_{2}(t) t^{p}-1=0 .
$$

Then the sequence $\left\{x_{n}\right\}$ generated by 1.2 for $x_{0} \in U\left(x^{*}, r_{2}\right)-\left\{x^{*}\right\}$ is well defined, remains in $U\left(x^{*}, r_{2}\right)$ for each $n=0,1,2, \ldots$ and converges to $x^{*}$. Moreover,

$$
\left\|x_{n+1}-x^{*}\right\| \leq h_{2}\left(\left\|x_{n}-x^{*}\right\|\right)\left\|x_{n}-x^{*}\right\|^{p+1}<\left\|x_{n}-x^{*}\right\|<r_{2} .
$$

Proof. We have $h^{*}(0)=-1<0$ and $h^{*}(t) \rightarrow \infty$ as $t \rightarrow \infty$. Hence the intermediate value theorem implies that function $h^{*}$ has positive zeros. Denote by $r_{2}$ the smallest such zero. By hypothesis $x_{0} \in U\left(x^{*}, r_{2}\right)-\left\{x^{*}\right\}$. Then

$$
\begin{aligned}
\left\|x_{1}-x^{*}\right\| & =\left\|x_{0}-x^{*}-\left(A(F)\left(x_{0}\right)\right)^{-1} F\left(x_{0}\right)\right\| \\
& =\left\|\left(A(F)\left(x_{0}\right)\right)^{-1}\left[F\left(x^{*}\right)-F\left(x_{0}\right)-A(F)\left(x_{0}\right)\left(x^{*}-x_{0}\right)\right]\right\| \\
& \leq h_{2}\left(\left\|x_{0}-x^{*}\right\|\right)\left\|x_{0}-x^{*}\right\|^{p+1}<h_{2}\left(r_{2}\right) r_{2}^{p}\left\|x_{0}-x^{*}\right\| \\
& =\left\|x_{0}-x^{*}\right\|<r_{2},
\end{aligned}
$$

which shows that $x_{1} \in U\left(x^{*}, r_{2}\right)$ and $x_{2}$ is well defined. By a simple inductive argument as in the preceding estimate we get

$$
\begin{aligned}
\| x_{k+1} & -x^{*}\|=\| x_{k}-x^{*}-\left(A(F)\left(x_{k}\right)\right)^{-1} F\left(x_{k}\right) \| \\
& \leq\left\|\left(A(F)\left(x_{k}\right)\right)^{-1}\left[F\left(x^{*}\right)-F\left(x_{k}\right)-A(F)\left(x_{k}\right)\left(x^{*}-x_{k}\right)\right]\right\| \\
& \leq h_{2}\left(\left\|x_{k}-x^{*}\right\|\right)\left\|x_{k}-x^{*}\right\|^{p+1}<h_{2}\left(r_{2}\right) r_{2}^{p}\left\|x_{k}-x^{*}\right\|=\left\|x_{k}-x^{*}\right\|<r_{2},
\end{aligned}
$$

which shows $\lim _{k \rightarrow \infty} x_{k}=x^{*}$ and $x_{k+1} \in U\left(x^{*}, r_{2}\right)$.

REMARK 2.5. (a) Hypothesis 2.3 specializes to a Newton-Mysowskitype condition if $A(F)(x)=F^{\prime}(x)[7,12,16$. However, if $F$ is not Fréchetdifferentiable, then our results extend the applicability of 1.2 . 
(b) Theorem 2.1 has practical value despite the fact that we do not show that $x^{*}$ is a solution of $F(x)=0$, since this may be shown in another way.

(c) Hypothesis 2.12 can be replaced by the stronger

$$
\left\|(A(F)(x))^{-1}[F(x)-F(y)-A(F)(x)(x-y)]\right\| \leq h_{2}(\|x-y\|)\|x-y\|^{p+1} .
$$

The preceding results can be extended to two-point iterative algorithms defined for each $n=0,1,2, \ldots$ by

$$
x_{n+1}=x_{n}-\left(A(F)\left(x_{n}, x_{n-1}\right)\right)^{-1} F\left(x_{n}\right),
$$

where $x_{-1}, x_{0} \in D$ are initial points and $A(F)(w, v) \in L(X, Y)$ for each $v, w \in D$. If $A(F)(w, v)=[w, v ; F]$, then the iterative algorithm (2.13) reduces to the popular secant method, where $[w, v ; F]$ denotes a divided difference of order one for the operator $F$. Many other choices for $A$ are also possible [7, 12, 16].

If we simply replace $A(F)(x)$ by $A(F)(y, x)$ in the proof of Proposition 2.2, we arrive at the following semilocal convergence result for (2.13).

THEOREM 2.6. Let $F: D \subset X \rightarrow Y$ be a continuous operator and let $A(F)(y, x) \in L(X, Y)$ for each $x, y \in D$. Suppose that there exist $x_{-1}, x_{0} \in D$, $\eta \geq 0, p \geq 1, \psi>0$, and a function $h_{1}:[0, \eta] \rightarrow[0, \infty)$ continuous and nondecreasing such that for each $x, y \in D$,

$$
\begin{aligned}
& (A(F)(y, x))^{-1} \in L(Y, X), \quad\left\|(A(F)(y, x))^{-1}\right\| \leq \psi, \\
& \min \left\{\left\|x_{0}-x_{-1}\right\|,\left\|\left(A(F)\left(x_{0}, x_{-1}\right)\right)^{-1} F\left(x_{0}\right)\right\|\right\} \leq \eta \\
& \|F(y)-F(x)-A(F)(y, x)(y-x)\| \leq \frac{h_{1}(\|x-y\|)}{\psi}\|x-y\|^{p+1}, \\
& q_{1}<1, \quad q_{1} r_{1}^{p}<1 \\
& \bar{U}\left(x_{0}, r_{1}\right) \subseteq D
\end{aligned}
$$

where

$$
r_{1}=\frac{\eta}{1-q_{1}}
$$

and $q_{1}$ is defined in Proposition 2.2. Then the sequence $\left\{x_{n}\right\}$ generated by 2.13 is well defined, remains in $U\left(x_{0}, r_{1}\right)$ for each $n=0,1,2, \ldots$ and converges to the only solution of $F(x)=0$ in $\bar{U}\left(x_{0}, r_{1}\right)$. Moreover, the estimates (2.7) and (2.8) hold with $h_{1}$ and $q_{1}$ replacing $h$ and $q$, respectively.

Concerning, the local convergence of the iterative algorithm 2.13) we obtain a result analogous to Proposition 2.4.

Proposition 2.7. Let $F: D \subset X \rightarrow Y$ be a continuous operator and let $A(F)(y, x) \in L(X, Y)$. Suppose that there exist $x^{*} \in D, p \geq 1$, and a function $h_{2}:[0, \infty)^{2} \rightarrow[0, \infty)$ continuous and nondecreasing such that for 
each $x, y \in D$,

$$
\begin{aligned}
& F\left(x^{*}\right)=0, \quad(A(F)(y, x))^{-1} \in L(Y, X), \\
& \left\|(A(F)(y, x))^{-1}\left[F(y)-F\left(x^{*}\right)-A(F)(y, x)\left(y-x^{*}\right)\right]\right\| \\
& \leq h_{2}\left(\left\|y-x^{*}\right\|,\left\|x-x^{*}\right\|\right)\left\|y-x^{*}\right\|^{p+1} \\
& \bar{U}\left(x^{*}, r_{2}\right) \subseteq D,
\end{aligned}
$$

where $r_{2}$ is the smallest positive solution of

$$
h^{* *}(t):=h_{2}(t, t) t^{p}-1=0 .
$$

Then the sequence $\left\{x_{n}\right\}$ generated by 2.13) for $x_{-1}, x_{0} \in U\left(x^{*}, r_{2}\right)-\left\{x^{*}\right\}$ is well defined, remains in $U\left(x^{*}, r_{2}\right)$ for each $n=0,1,2, \ldots$ and converges to $x^{*}$. Moreover,

$$
\begin{aligned}
\left\|x_{n+1}-x^{*}\right\| & \leq h_{2}\left(\left\|x_{n}-x^{*}\right\|,\left\|x_{n-1}-x^{*}\right\|\right)\left\|x_{n}-x^{*}\right\|^{p+1} \\
& <\left\|x_{n}-x^{*}\right\|<r_{2} .
\end{aligned}
$$

REMARK 2.8. In the next section, we present some choices and properties of operators $A(F)(y, x)$ satisfying the crucial estimate $(2.15)$. In particular, we choose

$$
\begin{array}{ll}
h_{1}(t)=\frac{\rho \psi t}{p} \quad \text { for some } \rho>0(\text { see }(3.14)), \\
\left.h_{1}(t)=\frac{c \psi}{(p+1) !} \quad \text { for some } c>0(\text { see } 3.27)\right), \\
h_{1}(t)=\frac{K \psi \gamma^{p+1}}{(p+1) !} \quad(\text { see }(3.49))
\end{array}
$$

if $|g(x)-g(y)| \leq \gamma|x-y|$ for some $K>0$ and $\gamma>0$.

Hence, Theorem 2.6 can be applied to solve the equation $F(x)=0$. Other choices for $A(\overline{F)}(x)$ or $A(F)(y, x)$ can be found in [5-7], [10-17].

3. Applications to fractional and vector calculi. We want to solve numerically

$$
f(x)=0 .
$$

(I) Application to fractional calculus. Let $p \in \mathbb{N}-\{1\}$ be such that $p-1<\nu<p$, where $\nu \notin \mathbb{N}, \nu>0$, i.e. $\lceil\nu\rceil=p, a<b, f \in C^{p}([a, b])$.

We define the following left Caputo fractional derivatives (see [3, p. 270]):

$$
\left(D_{* y}^{\nu} f\right)(x):=\frac{1}{\Gamma(p-\nu)} \int_{y}^{x}(x-t)^{p-\nu-1} f^{(p)}(t) d t
$$

when $x \geq y$, and 


$$
\left(D_{* x}^{\nu} f\right)(y):=\frac{1}{\Gamma(p-\nu)} \int_{x}^{y}(y-t)^{p-\nu-1} f^{(p)}(t) d t,
$$

when $y \geq x$, where $\Gamma$ is the gamma function.

We also define the linear operator

$$
\left(A_{1}(f)\right)(x, y):= \begin{cases}\sum_{k=1}^{p-1} \frac{f^{(k)}(y)}{k !}(x-y)^{k-1}+\left(D_{* y}^{\nu} f\right)(x) \frac{(x-y)^{\nu-1}}{\Gamma(\nu+1)}, & x>y, \\ \sum_{k=1}^{p-1} \frac{f^{(k)}(x)}{k !}(y-x)^{k-1}+\left(D_{* x}^{\nu} f\right)(y) \frac{(y-x)^{\nu-1}}{\Gamma(\nu+1)}, & y>x, \\ f^{(p-1)}(x), & x=y .\end{cases}
$$

By the left fractional Caputo Taylor formula (see [8, p. 54] and [3, p. 395]), we get

$$
\begin{aligned}
f(x)-f(y)= & \sum_{k=1}^{p-1} \frac{f^{(k)}(y)}{k !}(x-y)^{k} \\
& +\frac{1}{\Gamma(\nu)} \int_{y}^{x}(x-t)^{\nu-1} D_{* y}^{\nu} f(t) d t \quad \text { for } x>y,
\end{aligned}
$$

and

$$
\begin{aligned}
f(y)-f(x)= & \sum_{k=1}^{p-1} \frac{f^{(k)}(x)}{k !}(y-x)^{k} \\
& +\frac{1}{\Gamma(\nu)} \int_{x}^{y}(y-t)^{\nu-1} D_{* x}^{\nu} f(t) d t \quad \text { for } x<y .
\end{aligned}
$$

We immediately observe that

$$
\begin{aligned}
\left|\left(A_{1}(f)\right)(x, x)-\left(A_{1}(f)\right)(y, y)\right| & =\left|f^{(p-1)}(x)-f^{(p-1)}(y)\right| \\
& \leq\left\|f^{(p)}\right\|_{\infty}|x-y|, \quad \forall x, y \in[a, b],
\end{aligned}
$$

We would like to prove that

$$
\left|f(x)-f(y)-\left(A_{1}(f)\right)(x, y)(x-y)\right| \leq c \frac{|x-y|^{p}}{p}
$$

for any $x, y \in[a, b]$ and some constant $0<c<1$.

For $x=y,(3.8)$ is trivial.

We assume $x \neq y$. We distinguish two cases:

If $x>y$, we observe that

$$
\begin{aligned}
& \left|f(x)-f(y)-\left(A_{1}(f)\right)(x, y)(x-y)\right| \\
& =\mid \sum_{k=1}^{p-1} \frac{f^{(k)}(y)}{k !}(x-y)^{k}+\frac{1}{\Gamma(\nu)} \int_{y}^{x}(x-t)^{\nu-1} D_{* y}^{\nu} f(t) d t \\
& \quad-\sum_{k=1}^{p-1} \frac{f^{(k)}(y)}{k !}(x-y)^{k}-\left(D_{* y}^{\nu} f\right)(x) \frac{(x-y)^{\nu}}{\Gamma(\nu+1)} \mid
\end{aligned}
$$




$$
\begin{aligned}
& =\left|\frac{1}{\Gamma(\nu)} \int_{y}^{x}(x-t)^{\nu-1}\left(D_{* y}^{\nu} f\right)(t) d t-\frac{1}{\Gamma(\nu)} \int_{y}^{x}(x-t)^{\nu-1}\left(D_{* y}^{\nu} f\right)(x) d t\right| \\
& =\frac{1}{\Gamma(\nu)}\left|\int_{y}^{x}(x-t)^{\nu-1}\left(\left(D_{* y}^{\nu} f\right)(t)-\left(D_{* y}^{\nu} f\right)(x)\right) d t\right| \\
& \leq \frac{1}{\Gamma(\nu)} \int_{y}^{x}(x-t)^{\nu-1}\left|\left(D_{* y}^{\nu} f\right)(t)-\left(D_{* y}^{\nu} f\right)(x)\right| d t
\end{aligned}
$$

(we note that $\left|\left(D_{* y}^{\nu} f\right)(t)-\left(D_{* y}^{\nu} f\right)(x)\right| \leq \lambda_{1}|t-x|^{p-\nu}$ for any $t, x, y \in[a, b]$ with $x \geq t \geq y$, where $\lambda_{1}<\Gamma(\nu)$, i.e. $\left.\rho_{1}:=\lambda_{1} / \Gamma(\nu)<1\right)$

$$
\begin{aligned}
& \leq \frac{\lambda_{1}}{\Gamma(\nu)} \int_{y}^{x}(x-t)^{\nu-1}(x-t)^{p-\nu} d t \\
& =\frac{\lambda_{1}}{\Gamma(\nu)} \int_{y}^{x}(x-t)^{p-1} d t=\frac{\lambda_{1}}{\Gamma(\nu)} \frac{(x-y)^{p}}{p}=\rho_{1} \frac{(x-y)^{p}}{p} .
\end{aligned}
$$

We have proved that

$$
\left|f(x)-f(y)-\left(A_{1}(f)\right)(x, y)(x-y)\right| \leq \rho_{1} \frac{(x-y)^{p}}{p}
$$

for $x>y$, where $0<\rho_{1}<1$. If $x<y$, we observe that

$$
\begin{aligned}
& =\left|f(y)-f(x)-\left(A_{1}(f)\right)(x, y)(y-x)\right| \\
& =\mid \sum_{k=1}^{p-1} \frac{f^{(k)}(x)}{k !}(y-x)^{k}+\frac{1}{\Gamma(\nu)} \int_{x}^{y}(y-t)^{\nu-1}\left(D_{* x}^{\nu} f\right)(t) d t \\
& \quad-\sum_{k=1}^{p-1} \frac{f^{(k)}(x)}{k !}(y-x)^{k}-\left(D_{* x}^{\nu} f\right)(y) \frac{(y-x)^{\nu}}{\Gamma(\nu+1)} \mid \\
& =\left|\frac{1}{\Gamma(\nu)} \int_{x}^{y}(y-t)^{\nu-1}\left(D_{* x}^{\nu} f\right)(t) d t-\frac{1}{\Gamma(\nu)} \int_{x}^{y}(y-t)^{\nu-1}\left(D_{* x}^{\nu} f\right)(y) d t\right|
\end{aligned}
$$$$
=\frac{1}{\Gamma(\nu)}\left|\int_{x}^{y}(y-t)^{\nu-1}\left(\left(D_{* x}^{\nu} f\right)(t)-\left(D_{* x}^{\nu} f\right)(y)\right) d t\right|
$$$$
\leq \frac{1}{\Gamma(\nu)} \int_{x}^{y}(y-t)^{\nu-1}\left|\left(D_{* x}^{\nu} f\right)(t)-\left(D_{* x}^{\nu} f\right)(y)\right| d t
$$

(we note that $\left|\left(D_{* x}^{\nu} f\right)(t)-\left(D_{* x}^{\nu} f\right)(y)\right| \leq \lambda_{2}|t-y|^{p-\nu}$ for any $t, y, x \in[a, b]$ with $y \geq t \geq x)$ 


$$
\begin{aligned}
& \leq \frac{\lambda_{2}}{\Gamma(\nu)} \int_{x}^{y}(y-t)^{\nu-1}(y-t)^{p-\nu} d t \\
& =\frac{\lambda_{2}}{\Gamma(\nu)} \int_{x}^{y}(y-t)^{p-1} d t=\frac{\lambda_{2}}{\Gamma(\nu)} \frac{(y-x)^{p}}{p} .
\end{aligned}
$$

Assuming also

$$
\rho_{2}:=\lambda_{2} / \Gamma(\nu)<1
$$

(i.e. $\lambda_{2}<\Gamma(\nu)$ ), we have proved that

$$
\left|f(x)-f(y)-\left(A_{1}(f)\right)(x, y)(x-y)\right| \leq \rho_{2} \frac{(y-x)^{p}}{p} \quad \text { for } x<y .
$$

Conclusion. If $\lambda:=\max \left(\lambda_{1}, \lambda_{2}\right)$ and $\rho:=\lambda / \Gamma(\nu)<1$, then

$$
\left|f(x)-f(y)-\left(A_{1}(f)\right)(x, y)(x-y)\right| \leq \rho \frac{|x-y|^{p}}{p} \quad \text { for any } x, y \in[a, b] .
$$

\section{(II) Application to vector calculus}

(II $\left.{ }_{1}\right)$ Background (see [19, pp. 83-94]). Let $f(t)$ be a function defined on $[a, b] \subseteq \mathbb{R}$ taking values in a real or complex normed linear space $(X,\|\cdot\|)$. The derivatives of $f$ here are defined just as for numerical functions. For all the properties of derivatives see [19, pp. 83-86].

From now let $(X,\|\cdot\|)$ be a Banach space and $f:[a, b] \rightarrow X$.

We define the vector valued Riemann integral $\int_{a}^{b} f(t) d t \in X$ as the limit of the vector valued Riemann sums in $X$, with convergence in $\|\cdot\|$. The definition is as for numerical functions.

If $\int_{a}^{b} f(t) d t \in X$ we call $f$ integrable on $[a, b]$. If $f \in C([a, b], X)$, then $f$ is integrable [19, p. 87].

For other properties of vector valued Riemann integrals see [19, pp. 8691]. We mention some of them here.

Let $f, g$ be vector valued Riemann integrable functions. We have (see [19, p. 88])

$$
\begin{aligned}
& \int_{a}^{b} \alpha f(t) d t=\alpha \int_{a}^{b} f(t) d t, \quad \alpha \in \mathbb{R} \text { or } \alpha \in \mathbb{C}, \\
& \int_{a}^{b}(f(t)+g(t)) d t=\int_{a}^{b} f(t) d t+\int_{a}^{b} g(t) d t, \\
& \int_{a}^{c} f(t) d t+\int_{c}^{b} f(t) d t=\int_{a}^{b} f(t) d t, \quad a<c<b,
\end{aligned}
$$




$$
\begin{aligned}
& \left\|\int_{a}^{b} f(t) d t\right\| \leq(b-a) \max _{a \leq t \leq b}\|f(t)\|, \\
& \left\|\int_{a}^{b} f(t) d t\right\| \leq \int_{a}^{b}\|f(t)\| d t .
\end{aligned}
$$

We also set by convention

$$
\int_{\alpha}^{\beta} f(t) d t=-\int_{\beta}^{\alpha} f(t) d t \quad \text { for } a \leq \beta \leq \alpha \leq b .
$$

Let $f:[a, b] \rightarrow \mathbb{R}$ be a Riemann integrable function, i.e. $\int_{a}^{b} f(t) d t$ exists as a real number, and $c \in X$. Then clearly

$$
c \int_{a}^{b} f(x) d x=\int_{a}^{b} c f(x) d x \in X .
$$

We define the space $C^{p}([a, b], X), p \in \mathbb{N}$, of $p$-times continuously differentiable functions from $[a, b]$ into $X$; here continuity is with respect to $\|\cdot\|$ and defined in the usual way as for numerical functions.

Let $(X,\|\cdot\|)$ be a Banach space and $f \in C^{p}([a, b], X)$. Then we have the vector valued Taylor formula (see [19, pp. 93-94] and also [18, (IV, 9; 47)]

$$
\begin{array}{r}
f(y)-f(x)-f^{\prime}(x)(y-x)-\frac{1}{2} f^{\prime \prime}(x)(y-x)^{2}-\cdots-\frac{1}{(p-1) !} f^{(p-1)}(x)(y-x)^{p-1} \\
=\frac{1}{(p-1) !} \int_{x}^{y}(y-t)^{p-1} f^{(p)}(t) d t, \quad \forall x, y \in[a, b] .
\end{array}
$$

In particular 3.22 is true when $X=\mathbb{R}^{m}$ or $\mathbb{C}^{m}, m \in \mathbb{N}$. Clearly

$$
\begin{aligned}
& f(y)-f(x) \\
& =\sum_{k=1}^{p} \frac{f^{(k)}(x)}{k !}(y-x)^{k}+\frac{1}{(p-1) !} \int_{x}^{y}(y-t)^{p-1}\left(f^{(p)}(t)-f^{(p)}(x)\right) d t
\end{aligned}
$$

for all $x, y \in[a, b]$.

We will use (3.23).

We also need the mean value theorem for Banach space valued functions.

Theorem 3.1 (see [13, p. 3]). Let $f \in C([a, b], X)$, where $X$ is a Banach space. Assume $f^{\prime}$ exists on $[a, b]$ and $\left\|f^{\prime}(t)\right\| \leq K$ for $a<t<b$. Then

$$
\|f(b)-f(a)\| \leq K(b-a) .
$$


(II 2$)$ From now on we assume that $f \in C^{p+1}([a, b], X), p \in \mathbb{N}$. We define

$$
\left(A_{2}(f)\right)(x, y):= \begin{cases}\sum_{k=1}^{p} \frac{f^{(k)}(x)}{k !}(y-x)^{k-1}, & x \neq y \\ f^{(p)}(x), & x=y .\end{cases}
$$

Then

$$
\begin{aligned}
\left\|\left(A_{2}(f)\right)(x, x)-\left(A_{2}(f)\right)(y, y)\right\| & =\left\|f^{(p)}(x)-f^{(p)}(y)\right\| \\
\leq & \left\|f^{(p+1)}\left|\|_{\infty}\right| x-y \mid, \quad \forall x, y \in[a, b],\right.
\end{aligned}
$$

where

$$
\left\|f^{(p+1)} \mid\right\|_{\infty}:=\sup _{t \in[a, b]}\left\|f^{(p+1)}(t)\right\|<\infty .
$$

We would like to prove that

$$
\left\|f(y)-f(x)-\left(A_{2}(f)\right)(x, y)(y-x)\right\| \leq c \frac{|x-y|^{p+1}}{(p+1) !},
$$

where $c>0$.

When $x=y$, inequality 3.27 is trivially true. We will prove it for $x \neq y$.

We observe that

$$
\begin{aligned}
& =\| \sum_{k=1}^{p} \frac{f^{(k)}(x)}{k !}(y-x)^{k}+\frac{1}{(p-1) !} \int_{x}^{y}(y-t)^{p-1}\left(f^{(p)}(t)-f^{(p)}(x)\right) d t \\
& -\sum_{k=1}^{p} \frac{f^{(k)}(x)}{k !}(y-x)^{k} \| \\
& =\frac{1}{(p-1) !}\left\|\int_{x}^{y}(y-t)^{p-1}\left(f^{(p)}(t)-f^{(p)}(x)\right) d t\right\|=:(\xi) .
\end{aligned}
$$

Let $y>x$. We observe that

$$
\begin{aligned}
(\xi) & \leq \frac{1}{(p-1) !} \int_{x}^{y}(y-t)^{p-1}\left\|f^{(p)}(t)-f^{(p)}(x)\right\| d t \\
& \leq \frac{\|\| f^{(p+1)} \mid \|_{\infty}}{(p-1) !} \int_{x}^{y}(y-t)^{p-1}(t-x)^{2-1} d t \\
& =\frac{\|\| f^{(p+1)} \mid \|_{\infty}}{(p-1) !} \frac{\Gamma(p) \Gamma(2)}{\Gamma(p+2)}(y-x)^{p+1} \\
& =\frac{\|\| f^{(p+1)} \mid \|_{\infty}}{(p-1) !} \frac{(p-1) !}{(p+1) !}(y-x)^{p+1}=\frac{\left\|f^{(p+1)}\right\| \|_{\infty}}{(p+1) !}(y-x)^{p+1} .
\end{aligned}
$$


Hence,

$$
(\xi) \leq \frac{\left\|\mid f^{(p+1)}\right\| \|_{\infty}}{(p+1) !}(y-x)^{p+1} \quad \text { for } y>x .
$$

Let now $x>y$. We observe that

$$
\begin{aligned}
(\xi) & =\frac{1}{(p-1) !}\left\|\int_{y}^{x}(y-t)^{p-1}\left(f^{(p)}(t)-f^{(p)}(x)\right) d t\right\| \\
& \leq \frac{1}{(p-1) !} \int_{y}^{x}(t-y)^{p-1}\left\|f^{(p)}(t)-f^{(p)}(x)\right\| d t \\
& \leq \frac{\left\|f^{(p+1)}\right\| \|_{\infty}}{(p-1) !} \int_{y}^{x}(x-t)^{2-1}(t-y)^{p-1} d t \\
& =\frac{\left\|f^{(p+1)}\right\| \|_{\infty}}{(p-1) !} \frac{\Gamma(p) \Gamma(2)}{\Gamma(p+2)}(x-y)^{p+1} \\
& =\frac{\left\|f^{(p+1)}\right\| \|_{\infty}}{(p-1) !} \frac{(p-1) !}{(p+1) !}(x-y)^{p+1}=\frac{\left\|f^{(p+1)}\right\|_{\infty}}{(p+1) !}(x-y)^{p+1} .
\end{aligned}
$$

We have proved that

$$
(\xi) \leq \frac{\left\|\mid f^{(p+1)}\right\| \|_{\infty}}{(p+1) !}(x-y)^{p+1} \quad \text { for } x>y .
$$

Conclusion. Let $p \in \mathbb{N}$ and $f \in C^{p+1}([a, b])$. Then

$$
\begin{aligned}
& \left\|f(y)-f(x)-\left(A_{2}(f)\right)(x, y) \cdot(y-x)\right\| \\
& \leq \frac{\left\|f^{(p+1)} \mid\right\|_{\infty}}{(p+1) !}|x-y|^{p+1}, \quad \forall x, y \in[a, b] .
\end{aligned}
$$

(III) Applications from mathematical analysis. In [4, pp. 400402], we have proved the following general Taylor formula:

THEOREM 3.2. Let $f, f^{\prime}, \ldots, f^{(p)}$ and $g, g^{\prime}$ be continuous from $[a, b]$ (or $[b, a])$ into $\mathbb{R}, p \in \mathbb{N}$. Assume $\left(g^{-1}\right)^{(k)}, k=0,1, \ldots, p$, are continuous. Then

$$
f(b)=f(a)+\sum_{k=1}^{p-1} \frac{\left(f \circ g^{-1}\right)^{(k)}(g(a))}{k !}(g(b)-g(a))^{k}+R_{p}(a, b),
$$

where

$$
\begin{aligned}
R_{p}(a, b) & =\frac{1}{(p-1) !} \int_{a}^{b}(g(b)-g(s))^{p-1}\left(f \circ g^{-1}\right)^{(p)}(g(s)) g^{\prime}(s) d s \\
& =\frac{1}{(p-1) !} \int_{g(a)}^{g(b)}(g(b)-t)^{p-1}\left(f \circ g^{-1}\right)^{(p)}(t) d t
\end{aligned}
$$


Theorem 3.2 will be applied for $g(x)=e^{x}$. One can give similar applications for $g=\sin , \cos$, tan, etc., over suitable intervals (see [4, p. 402]).

Proposition 3.3. Let $f^{(p)}$ be continuous from $[a, b]($ or $[b, a])$ into $\mathbb{R}$, $p \in \mathbb{N}$. Then

$$
f(b)=f(a)+\sum_{k=1}^{p-1} \frac{(f \circ \ln )^{(k)}\left(e^{a}\right)}{k !}\left(e^{b}-e^{a}\right)^{k}+R_{p}(a, b),
$$

where

$$
\begin{aligned}
R_{p}(a, b) & =\frac{1}{(p-1) !} \int_{e^{a}}^{e^{b}}\left(e^{b}-t\right)^{p-1}(f \circ \ln )^{(p)}(t) d t \\
& =\frac{1}{(p-1) !} \int_{a}^{b}\left(e^{b}-e^{a}\right)^{p-1}(f \circ \ln )^{(p)}\left(e^{s}\right) \cdot e^{s} d s .
\end{aligned}
$$

We will use the following variant.

Theorem 3.4. Let $f, f^{\prime}, \ldots, f^{(p)}, p \in \mathbb{N}$, and $g, g^{\prime}$ be continuous from $[a, b]$ into $\mathbb{R}$. Assume that $\left(g^{-1}\right)^{(k)}, k=0,1, \ldots, p$, are continuous. Then

$$
f(\beta)-f(\alpha)=\sum_{k=1}^{p} \frac{\left(f \circ g^{-1}\right)^{(k)}(g(\alpha))}{k !}(g(\beta)-g(\alpha))^{k}+R_{p}^{*}(\alpha, \beta),
$$

where

$$
\begin{aligned}
& =\frac{1}{(p-1) !} \int_{\alpha}^{\beta}(g(\beta)-g(s))^{p-1}\left(\left(f \circ g^{-1}\right)^{(p)}(g(s))-\left(f \circ g^{-1}\right)^{(p)}(g(\alpha))\right) g^{\prime}(s) d s \\
& =\frac{1}{(p-1) !} \int_{g(\alpha)}^{g(\beta)}(g(\beta)-t)^{p-1}\left(\left(f \circ g^{-1}\right)^{(p)}(t)-\left(f \circ g^{-1}\right)^{(p)}(g(\alpha))\right) d t
\end{aligned}
$$

for all $\alpha, \beta \in[a, b]$.

\section{Proof. Easy.}

REMARK 3.5. Set $l=f \circ g^{-1}$. Then $l, l^{\prime}, \ldots, l^{(p)}$ are continuous from $g([a, b])$ into $f([a, b])$.

Next we estimate $R_{p}^{*}(\alpha, \beta)$. We assume that

$$
\left|\left(f \circ g^{-1}\right)^{(p)}(t)-\left(f \circ g^{-1}\right)^{(p)}(g(\alpha))\right| \leq K|t-g(\alpha)|
$$

for all $t, g(\alpha) \in[g(a), g(b)]$, or for all $t, g(\alpha) \in[g(b), g(a)]$, where $K>0$.

We distinguish two cases: 
(i) If $g(\beta)>g(\alpha)$, then

$$
\begin{aligned}
& \left|R_{p}^{*}(\alpha, \beta)\right| \\
& \leq \frac{1}{(p-1) !} \int_{g(\alpha)}^{g(\beta)}(g(\beta)-t)^{p-1}\left|\left(f \circ g^{-1}\right)^{(p)}(t)-\left(f \circ g^{-1}\right)^{(p)}(g(\alpha))\right| d t \\
& \leq \frac{K}{(p-1) !} \int_{g(\alpha)}^{g(\beta)}(g(\beta)-t)^{p-1}(t-g(\alpha))^{2-1} d t \\
& =\frac{K}{(p-1) !} \frac{\Gamma(p) \Gamma(2)}{\Gamma(p+2)}(g(\beta)-g(\alpha))^{p+1} \\
& =\frac{K}{(p-1) !} \frac{(p-1) !}{(p+1) !}(g(\beta)-g(\alpha))^{p+1}=K \frac{(g(\beta)-g(\alpha))^{p+1}}{(p+1) !} .
\end{aligned}
$$

We have proved that

$$
\left|R_{p}^{*}(\alpha, \beta)\right| \leq K \frac{(g(\beta)-g(\alpha))^{p+1}}{(p+1) !} \quad \text { when } g(\beta)>g(\alpha) .
$$

(ii) If $g(\alpha)>g(\beta)$, then

$$
\begin{aligned}
& \left|R_{p}^{*}(\alpha, \beta)\right| \\
= & \frac{1}{(p-1) !}\left|\int_{g(\beta)}^{g(\alpha)}(t-g(\beta))^{p-1}\left(\left(f \circ g^{-1}\right)^{(p)}(t)-\left(f \circ g^{-1}\right)^{(p)}(g(\alpha))\right) d t\right| \\
\leq & \frac{1}{(p-1) !} \int_{g(\beta)}^{g(\alpha)}(t-g(\beta))^{p-1}\left|\left(f \circ g^{-1}\right)^{(p)}(t)-\left(f \circ g^{-1}\right)^{(p)}(g(\alpha))\right| d t \\
\leq & \frac{K}{(p-1) !} \int_{g(\beta)}^{g(\alpha)}(g(\alpha)-t)^{2-1}(t-g(\beta))^{p-1} d t \\
= & \frac{K}{(p-1) !} \frac{\Gamma(2) \Gamma(p)}{\Gamma(p+2)}(g(\alpha)-g(\beta))^{p+1} \\
= & \frac{K}{(p-1) !} \frac{(p-1) !}{(p+1) !}(g(\alpha)-g(\beta))^{p+1}=K \frac{(g(\alpha)-g(\beta))^{p+1}}{(p+1) !} .
\end{aligned}
$$

We have proved that

$$
\left|R_{p}^{*}(\alpha, \beta)\right| \leq K \frac{(g(\alpha)-g(\beta))^{p+1}}{(p+1) !} \quad \text { when } g(\alpha)>g(\beta) .
$$

Conclusion. We have

$$
\left|R_{p}^{*}(\alpha, \beta)\right| \leq K \frac{|g(\alpha)-g(\beta)|^{p+1}}{(p+1) !}, \quad \forall \alpha, \beta \in[a, b] .
$$


Both sides of (3.45) equal zero when $\alpha=\beta$.

We define the following linear operator:

$$
\left(A_{3}(f)\right)(x, y):= \begin{cases}\sum_{k=1}^{p} \frac{\left(f \circ g^{-1}\right)^{(k)}(g(y))}{k !}(g(x)-g(y))^{k-1}, & g(x) \neq g(y), \\ f^{(p-1)}(x), & x=y,\end{cases}
$$

for any $x, y \in[a, b]$.

Easily, we see that

$$
\begin{aligned}
\left|\left(A_{3}(f)\right)(x, x)-\left(A_{3}(f)\right)(y, y)\right| & =\left|f^{(p-1)}(x)-f^{(p-1)}(y)\right| \\
& \leq\left\|f^{(p)}\right\|_{\infty}|x-y|, \quad \forall x, y \in[a, b] .
\end{aligned}
$$

Next we observe that if $g(x) \neq g(y)$ then

$$
\begin{aligned}
& =\mid \sum_{k=1}^{p} \frac{\left(f \circ g^{-1}\right)^{(k)}(g(y))}{k !}(g(x)-g(y))^{k}+R_{p}^{*}(y, x) \\
& \quad-\left(\sum_{k=1}^{p} \frac{\left(f \circ g^{-1}\right)^{(k)}(g(y))}{k !}(g(x)-g(y))^{k-1}(g(x)-g(y))\right) \mid \\
& =\left|R_{p}^{*}(y, x)\right| \stackrel{3.45}{\leq} K \frac{|g(x)-g(y)|^{p+1}}{(p+1) !} .
\end{aligned}
$$

We have proved that

$$
\left|f(x)-f(y)-\left(A_{3}(f)\right)(x, y) \cdot(g(x)-g(y))\right| \leq K \frac{|g(x)-g(y)|^{p+1}}{(p+1) !}
$$

for all $x, y \in[a, b]$ (the case $x=y$ is trivial).

We apply the above theory as follows:

(III $)_{1}$ We define

$$
\left(A_{31}(f)\right)(x, y):= \begin{cases}\sum_{k=1}^{p} \frac{(f \circ \ln )^{(k)}\left(e^{y}\right)}{k !}\left(e^{x}-e^{y}\right)^{k-1}, & x \neq y \\ f^{(p-1)}(x), & x=y\end{cases}
$$

for any $x, y \in[a, b]$. Then

$$
\left|f(x)-f(y)-\left(A_{31}(f)\right)(x, y) \cdot\left(e^{x}-e^{y}\right)\right| \leq K_{1} \frac{\left|e^{x}-e^{y}\right|^{p+1}}{(p+1) !}
$$

for all $x, y \in[a, b]$, where we assume that

$$
\left|(f \circ \ln )^{(p)}(t)-(f \circ \ln )^{(p)}\left(e^{y}\right)\right| \leq K_{1}\left|t-e^{y}\right|
$$

for all $t, e^{y} \in\left[e^{a}, e^{b}\right], a<b$, with $K_{1}>0$. 
$\left(\mathbf{I I I}_{2}\right)$ Next let $f \in C^{p}([-\pi / 2+\varepsilon, \pi / 2-\varepsilon]), p \in \mathbb{N}, \varepsilon>0$ small. We define

$$
\left(A_{32}(f)\right)(x, y):= \begin{cases}\sum_{k=1}^{p} \frac{\left(f \circ \sin ^{-1}\right)^{(k)}(\sin y)}{k !}(\sin x-\sin y)^{k-1}, & x \neq y, \\ f^{(p-1)}(x), & x=y,\end{cases}
$$

for any $x, y \in[-\pi / 2+\varepsilon, \pi / 2-\varepsilon]$.

We assume that

$$
\left|\left(f \circ \sin ^{-1}\right)^{(p)}(t)-\left(f \circ \sin ^{-1}\right)^{(p)}(\sin y)\right| \leq K_{2}|t-\sin y|
$$

for all $t, \sin y \in[\sin (-\pi / 2+\varepsilon), \sin (\pi / 2-\varepsilon)]$, where $K_{2}>0$. Then

$$
\left|f(x)-f(y)-\left(A_{32}(f)\right)(x, y) \cdot(\sin x-\sin y)\right| \leq K_{2} \frac{|\sin x-\sin y|^{p+1}}{(p+1) !}
$$

for all $x, y \in[-\pi / 2+\varepsilon, \pi / 2-\varepsilon]$.

(III $\left.{ }_{3}\right)$ Next let $f \in C^{p}([\varepsilon, \pi-\varepsilon]), p \in \mathbb{N}, \varepsilon>0$ small. We define

$$
\left(A_{33}(f)\right)(x, y):= \begin{cases}\sum_{k=1}^{p} \frac{\left(f \circ \cos ^{-1}\right)^{(k)}(\cos y)}{k !}(\cos x-\cos y)^{k-1}, & x \neq y \\ f^{(p-1)}(x), & x=y\end{cases}
$$

for any $x, y \in[\varepsilon, \pi-\varepsilon]$. We assume that

$$
\left|\left(f \circ \cos ^{-1}\right)^{(p)}(t)-\left(f \circ \cos ^{-1}\right)^{(p)}(\cos y)\right| \leq K_{3}|t-\cos y|
$$

for all $t, \cos y \in[\cos \varepsilon, \cos (\pi-\varepsilon)]$, where $K_{3}>0$. Then

$$
\left|f(x)-f(y)-\left(A_{33}(f)\right)(x, y) \cdot(\cos x-\cos y)\right| \leq K_{3} \frac{|\cos x-\cos y|^{p+1}}{(p+1) !}
$$
for all $x, y \in[\varepsilon, \pi-\varepsilon]$.

$\left(\mathbf{I I I}_{4}\right)$ Let $f \in C^{p}([-\pi / 2+\varepsilon, \pi / 2-\varepsilon]), p \in \mathbb{N}, \varepsilon>0$ small. We define

$$
\left(A_{34}(f)\right)(x, y):= \begin{cases}\sum_{k=1}^{p} \frac{\left(f \circ \tan ^{-1}\right)^{(k)}(\tan y)}{k !}(\tan x-\tan y)^{k-1}, & x \neq y \\ f^{(p-1)}(x), & x=y\end{cases}
$$

for any $x, y \in[-\pi / 2+\varepsilon, \pi / 2-\varepsilon]$. We assume that

$$
\left|\left(f \circ \tan ^{-1}\right)^{(p)}(t)-\left(f \circ \tan ^{-1}\right)^{(p)}(\tan y)\right| \leq K_{4}|t-\tan y|
$$

for all $t, \tan y \in[\tan (-\pi / 2+\varepsilon), \tan (\pi / 2-\varepsilon)]$, where $K_{4}>0$. Then

(3.61) $\left|f(x)-f(y)-\left(A_{34}(f)\right)(x, y) \cdot(\tan x-\tan y)\right| \leq K_{4} \frac{|\tan x-\tan y|^{p+1}}{(p+1) !}$ for all $x, y \in[-\pi / 2+\varepsilon, \pi / 2-\varepsilon]$. 


\section{References}

[1] S. Amat and S. Busquier, Third-order iterative methods under Kantorovich conditions, J. Math. Anal. Appl. 336 (2007), 243-261.

[2] S. Amat, S. Busquier and S. Plaza, Chaotic dynamics of a third-order Newton-type method, J. Math. Anal. Appl. 366 (2010), 164-174.

[3] G. Anastassiou, Fractional Differentiation Inequalities, Springer, New York, 2009.

[4] G. Anastassiou, Intelligent Mathematics: Computational Analysis, Springer, Heidelberg, 2011.

[5] I. K. Argyros, Newton-like methods in partially ordered linear spaces, J. Approx. Theory Appl. 9 (1993), 1-10.

[6] I. K. Argyros, Results on controlling the residuals of perturbed Newton-like methods on Banach spaces with a convergence structure, Southwest J. Pure Appl. Math. 1 (1995), 32-38.

[7] I. K. Argyros, Convergence and Applications of Newton-type Iterations, Springer, New York, 2008.

[8] K. Diethelm, The Analysis of Fractional Differential Equations, Lecture Notes in Math. 2004, Springer, New York, 2010.

[9] B. Driver, The Riemann Integral, www.math.ucsd.edu/ ${ }^{\circ}$ bdriver/231-02-03/Lecture _Notes/Chap4.pdf.

[10] J. A. Ezquerro, J. M. Gutiérrez, M. A. Hernández, N. Romero and M. J. Rubio, El método de Newton: de Newton a Kantorovich, Gac. R. Soc. Mat. Esp. 13 (2010), $53-76$.

[11] J. A. Ezquerro and M. A. Hernández, Newton-type methods of high order and domains of semilocal and global convergence, Appl. Math. Comput. 214, 1, (2009), 142-154.

[12] L. V. Kantorovich and G. P. Akilov, Functional Analysis in Normed Spaces, Pergamon Press, New York, 1964.

[13] G. Ladas and V. Lakshmikantham, Differential Equations in Abstract Spaces, Academic Press, New York, 1972.

[14] A. A. Magrenan, Different anomalies in a Jarratt family of iterative root finding methods, Appl. Math. Comput. 233 (2014), 29-38.

[15] A. A. Magrenan, A new tool to study real dynamics: The convergence plane, Appl. Math. Comput. 248 (2014), 215-224.

[16] F. A. Potra and V. Pták, Nondiscrete Induction and Iterative Processes, Pitman Publ., London, 1984.

[17] P. D. Proinov, New general convergence theory for iterative processes and its applications to Newton-Kantorovich type theorems, J. Complexity 26 (2010), 3-42.

[18] L. Schwartz, Analyse Mathématique, Hermann, Paris, 1967.

[19] G. Shilov, Elementary Functional Analysis, MIT Press, Cambridge, MA, 1974.

George A. Anastassiou

Department of Mathematical Sciences

University of Memphis

Memphis, TN 38152, U.S.A.

E-mail: ganastss@memphis.edu
Ioannis K. Argyros

Department of Mathematical Sciences

Cameron University

Lawton, OK 73505, U.S.A.

E-mail: iargyros@cameron.edu 